\title{
UK dentists' experience of iatrogenic trigeminal nerve injuries in relation to routine dental procedures: why, when and how often?
}

IN BRIEF
- The majority of dentists use Lidocaine
$2 \%$ for inferior dental blocks (IDBs) and
many are already using Articaine Buccal
infiltration technique.
The incidence of nerve injury related
to dental IDBs is 1 in 14,000 patients
undergoing routine dentistry and 1 in
3,300 undergoing care by specialists.
Notes the lack of knowledge by dentists
on where to seek advice and report
these injuries.

\author{
T. Renton, ${ }^{* 1}$ H. Janjua, ${ }^{2}$ J. E. Gallagher, ${ }^{3}$ M. Dalgleish ${ }^{4}$ and Z. Yilmaz ${ }^{5}$
}

VERIFIABLE CPD PAPER

Objectives To estimate the frequency of trigeminal nerve injuries associated with local anaesthetic administration, as experienced by UK dentists. Method A convenience sample of clinicians attending 12 study days over the UK was invited to complete an anonymised questionnaire exploring the nature of professional practice, dentists' practice demographics, experience of nerve injuries and related factors. Dental procedures related to reported trigeminal nerve injuries (TNIs) were divided into low risk, likely local anaesthetic (LA) related nerve injury, and high risk procedures, more likely to be direct nerve damage by the procedure (procedural related nerve injury). Data were analysed using Microsoft Excel and SPSS V17. Results Overall $79 \%$ of attendees completed a questionnaire $(n=415)$; clinicians held an average of 19 years' clinical experience. The numbers of clinicians surveyed included general dental practitioners $(n=290 ; 64 \%)$ and oral surgery (OS) specialists $(n=125 ; 36 \%)$. The estimated incidence of TNIs for the UK GDP workforce was 3,770 TNIs per year or 0.13 TNI per dentist per year. For specialists the incidence was increased to $0.39 \mathrm{TNI}$ per specialist per year. Of all injuries only half were reported, mainly to indemnity organisations. LA-related injuries were most common for GDPs and third molar surgery for OS specialists. It is estimated that TNIs will occur in 1 in 3,289 high risk procedures such as third molar surgery and 1 in 14,330 for low risk procedures, such as restorative dentistry, most likely LA-related. From 25\% to 29\% of these dental procedures related TNIs were permanent. Conclusions Nerve-related injuries in dentistry are not uncommon. Dental practitioners should be aware of the significant disability associated with iatrogenic nerve injuries and risk factors relating to LA-related trigeminal nerve injury. Clinicians should familiarise themselves with infiltration LA-techniques that may reduce these nerve injuries and with the Care Quality Commission regulations for reporting injuries to patients as a result of treatment.

\section{INTRODUCTION}

When we consider trigeminal nerve injuries (TNIs) in relation to dentistry we immediately think of high risk procedures including third molar surgery (TMS), implant and endodontic nerve injuries. ${ }^{1}$ Local anaesthetic (LA) related nerve injuries in relation to dentistry are often over looked and considered a rarity, though the incidence and prevalence remains unknown. The significance of LA-related TNIs should not be underestimated as there

\footnotetext{
Professor in Oral Surgery, ${ }^{2}$ Dental Student, ${ }^{3}$ Reader in Oral Health Services Research and Honorary Consultant in Dental Public Health, ${ }^{4}$ Project sponsor, ${ }^{5}$ Senior Scientist, Department Oral Surgery, Kings Health Parners Dental Institute, Kings College Hospital, Denmark Hill Campus, Bessemer Road, London, SE5 9RS *Correspondence to: Professor Tara Renton Email:Tara.renton@kcl.ac.uk
}

\section{Refereed Paper}

Accepted 5 April 2013

DOI: $10.1038 /$ sj.bdj.2013.583

${ }^{\circledR}$ British Dental Journal 2013; 214: 633-642 is no reparative treatment available, thus avoidance is preferable.

TNIs caused by local anaesthesia block injections have an estimated injury incidence of between $1: 26,762$ to $1 / 800,000 .^{2}$ Reports of incidences include 1:588,000 for prilocaine and 1/440,000 for articaine inferior alveolar nerve (IAN) blocks, both of which are 20 times greater than for Lidocaine injections. ${ }^{3,4}$ The nerve that is usually damaged during IAN block injections is the lingual nerve (LN), accounting for 70\% of nerve injuries. ${ }^{5}$ Recovery is reported to take place within eight weeks for $85-94 \%$ of cases $^{6}$ meaning that for about $10 \%$ of patients, injuries will be permanent. Large population surveys need to be carried out in order to determine the true incidence. ${ }^{3,4,7}$

Nerve injury due to LA is multi-factorial, with physical (needle, compression due to epineural or perineural haemorrhage) or chemical (haemorrhage or LA contents) components. The difference in fascicular structure of the $\mathrm{LN}^{8}$ and IAN at the region of the mandibular lingula is suggested to explain the increased likelihood of LN injuries in relation to injury during LA. ${ }^{2}$ A combination of peri-, epi- and intraneural trauma caused by haemorrhage, inflammation and scarring is likely to result in demyelination and consequently nerve injury. ${ }^{8}$ Most papers have focused on the fact that the technique and needle type may influence direct mechanical trauma to the nerve by the needle. ${ }^{8}$ The direct technique involving impacting the bone with the needle before emptying cartridge and then withdrawal of needle may cause additional bur deformation at the needle tip thus 'ripping' the nerve tissue. ${ }^{8}$ It is reported that $1.3-8.6 \%$ of patients experience an 'electric shock' type during IAN blocks and, as a result, 57\% of those 
patients suffer from prolonged neuropathy. ${ }^{2}$ Interestingly, it has been reported that $81 \%$ of IAN block nerve injuries resolve at two weeks post injection. ${ }^{4}$

Aside from mechanical causes, specific chemical agents and the LA components (type of agent, agent concentration, buffer, preservative) also play a potential role in nerve injury. ${ }^{9-12}$ In the UK several LA agents are available to dentists including; $2 \%$ lignocaine, 2\% mepivacaine, 3\% mepivacaine, $3 \%$ prilocaine, $4 \%$ prilocaine and $4 \%$ articaine. ${ }^{11}$ A study carried out by Perez-Castro et al. in $2009^{10}$ provided evidence that the concentration of the LA agent relates to persistent neuropathy, whereby their experiments showed that increasing concentration of LA agent significantly affected the survival rate of neurons in vitro. A recent report suggests that it is the type of anaesthetic that dictates the degree of inflammatory reaction to the LA, with lignocaine being the least irritant followed by articaine, mepivacaine and bupivicaine. ${ }^{13}$ The increased incidence of persistent nerve injury related to IAN blocks with the introduction of high concentration local anaesthetics (prilocaine and articaine both 4\%) has been highlighted by several epidemiological reports. $^{2-5}$

Articaine is said to have a number of advantages, namely low toxicity subsequent to inadvertent intravascular injection, ${ }^{14}$ which may be due to the rapid breakdown to an inactive metabolite (Articainic acid), rapid onset of surgical analgesia ( $2.5=/-1.1$ minutes $)$ compared with conventional lignocaine, ${ }^{15,16}$ and better diffusion through soft and hard tissue. ${ }^{17}$ There are, however, no significant benefits of using articaine 4\% compared with lignocaine $2 \%$ for IAN blocks. ${ }^{13,18}$ Septocaine components only differ in the active LA content and concentration. It is not yet conclusive whether this agent is more likely to induce permanent nerve injury. Several recent studies have investigated the potential neurotoxicity of commonly used LA agents. ${ }^{13,19,20}$ More recently, Hillerup et al. $^{21}$ have clearly demonstrated the toxicity of higher concentration LA agents to the IAN in vivo.

Management of patients with LA-related TNIs is mainly therapeutic, including pain relief for the $70 \%$ of patients experiencing

\section{Your Practice}

01 When did you qualify? Year of qualification.

Are you practising dentistry Yes $\square$ No $\square$ (if NO, please do not continue with further questions)

Q2 Type of clinical practice. Are you in:

General practice Yes $\square$ No $\square$ Specialty practice Yes $\square$ No $\square$

If yes please indicate:

Cosmetic $\square$ perio restorative $\square$ orthodontic $\square$ oral surgery $\square$ endo $\square$ paed $\square$ DCP: Therapist $\square$ Hygienist $\square$ Other $\square$ Please specify

03 Activity in practice. Are you (please tick):

Full time Yes $\square$ No $\square$ Part time Yes $\square$ No $\square$ If Yes what percentage...............\% What mix of activity do you do? ............... NHS N ..............\% Private

Q4 Approximately how many patients do you see/treat per year? Patients per year Trigeminal nerve injuries

05 How many nerve injuries have occurred in your patients, that are you are aware of, during your clinical experience? (please specify the number of cases)
$\begin{array}{lc}\text { None } \quad 1 & 2 \\ \text { If none go to question } 12 .\end{array}$

Q6 How many nerve injuries have occurred in your patients, that you are aware of, in the last year?

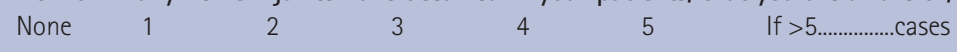

\begin{tabular}{|l|l|l|l|l|l|}
\hline Please insert $\checkmark$ where appropriate & \multicolumn{4}{|l|}{ Cases (specify for each case) } \\
\hline Q7 What procedures were these injuries related to? & 1 & 2 & 3 & 4 & 5 \\
\hline Restorative dentistry & & & & & \\
\hline Extraction of wisdom tooth & & & & & \\
\hline Extraction of other tooth close to nerve & & & & & \\
\hline Implant treatment & & & & & \\
\hline Endodontic therapy & & & & & \\
\hline Other please specify & & & & & \\
\hline Q8 Were the nerve injuries \\
temporary, <3months, permanent, $>3$ months?
\end{tabular}

Q11 Specific questions relating to Local anaesthetic practice

For Inferior dental blocks (IANBs) what LA do you most commonly use? Please tick one only Lignocaine 2\% $\square \quad$ Articaine 4\% $\square \quad$ Mepivocaine 3\% $\square \quad$ Prilocaine 2\% $\square \quad$ Prilocaine 3\% $\square$ Other $\square$ specifiy

If you use Articaine occasionally for IANBs what are the indications?

Repeat IANB / Other

Do you avoid using IANBS by using Articaine infiltration only technique? Yes $\square$ No $\square$ If you have had LA nerve injuries related to LA. Were they?

Related to multiple IANBs Yes $\square$ No $\square$ Related to Articaine Yes $\square \quad$ No $\square$ Pain on injection Yes $\square$ No $\square$

Lingual (case number.........) or Inferior alveolar nerve (case number.........)

Q12. Final comments

If you have any clinical experiences, views or questions about this survey please contact Tara Renton.

E-mail: tara.renton@kcl.ac.uk. Dept Oral Surgery, 4th floor KCL Dental Institute, London SE5 9RS

Thank you for taking time to complete this questionnaire

Fig. 1 Questionnaire used for LA nerve injury survey 


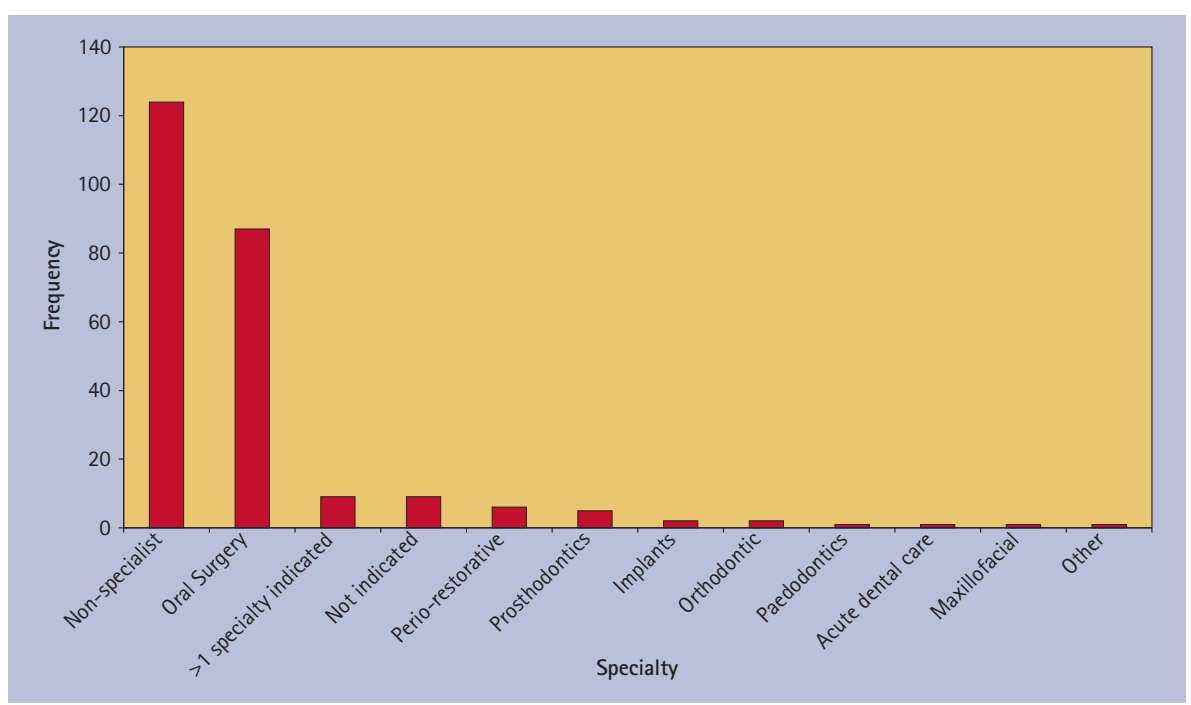

Fig. 2 Number of responders in primary dental practice and specialist care $(n=248)$ Nine dentists indicated that they specialised in more than one area (as indicated by the column labelled '>1 specialty')

chronic neuropathic pain connected to TNIs in relation to dentistry. ${ }^{22}$ The problem with these injuries is that the nerve will remain grossly intact and surgery is not indicated as one cannot identify the injured region. Studies have indicated a higher incidence of neuropathic pain among patients with post-traumatic trigeminal nerve injuries compared to other causes of peripheral nerve injury, ranging from $34 \%$ to $70 \% .^{1,12,23,24}$ Patients in pain with permanent nerve injuries are more likely to seek tertiary referral advice compared to patients with temporary and anaesthetic nerve injuries. ${ }^{1,22,23}$ Permanent chronic pain caused by LA sensory nerve injury will have a significant impact on those patients affected.

Currently the incidence and prevalence of dental LA-related nerve injuries is unclear. This study aimed to assess the prevalence and incidence of dental LA-related trigeminal nerve injuries in relation to routine dental procedures by surveying a convenience sample of UK GDPs and specialists; from attendees at 12 UK study days.

\section{METHODS}

In order to determine the rate of LA-related TNIs (defined as symptoms lasting seven days or more) experienced by UK dentists when providing dental procedures, we undertook a prospective sample method of questioning UK practising dentists who attended CPD meetings and oral surgery specialists attending their association's annual conference.
The questionnaire instrument, informed by the available literature, was developed to assess the incidence of trigeminal nerve injuries related to the LA administered by GDPs and specialists. The working group involved a dental public health academic/consultant assessment and patient input. The questionnaire (Fig. 1) included 13 questions about the nature of their clinical practice, demography, experience of nerve injuries and related factors. The questionnaire was piloted and refined using hospital clinicians. Dental procedures related to reported TNIs were divided into low risk (likely LA-related nerve injury - restorative) and high risk procedures more likely to be direct nerve damage by the procedure (less likely to be LA-related and more likely procedural related - third molar surgery [TMS], endodontics and implant surgery).

The rate was intended to be expressed as a number per million $\left(10^{6}\right)$ of relevant procedures:

1. The resultant rates were expected to be in the range 1 to 1,000 per million

2. There are 22,000 active dentists at approximately 5,000 practices $^{25}$ in the UK

3. There are approximately 800 oral surgery specialists registered with the GDC. ${ }^{25}$

The full time and part time working rates, specialism, NHS or private practice activity are currently unknown on any central UK database.

\section{ANALYSIS}

The data were manually entered on to computer and analysed using Microsoft Office Excel and the Statistical Package for the Social Sciences (SPSS) Version 17.0 statistical programme. Analysis of the incidence of TNIs reported by GDPs and specialists and subsequent actions were assessed using parametric and nonparametric methods of analyses as appropriate. The value of $\mathrm{p} \leq .05$ was chosen as level of statistical significance.

\section{RESULTS}

\section{Questionnaire participants}

In total, 415 conference participants completed questionnaires across the 12 professional meetings (79\% response rate). The average year of qualification was 1995 (mean 1994.56 for GDPs and 1995.55 for specialists; therefore average duration of career to date for GDPs was 19 years and 18 years for specialists). The majority at $99.5 \%$ were practising dentistry, of whom 290 (69\%) dentists were in general practice and 125 (30.1\%) in specialist practice.

Full time practice was being undertaken by 59\% GDPs and 56.8\% specialists. The majority of specialists were oral surgeons; nine dentists indicated that they specialised in more than one area (Fig. 2) and others indicated they were specialists in other fields ranging from perio-restorative dentistry, implants, orthodontics, paedodontics, acute dental care and maxillofacial surgery.

GDPs saw significantly more patients than specialists ( $p<0.001)$, whereby the average number of patients seen per year was 2,457 by GDPs compared to 1,283 by specialists.

\section{Incidence of injuries}

The numbers of TNIs experienced by all clinicians over their career and in the last year are illustrated in Table 1. When questioned regarding the number of nerve injuries that respondents were aware of having occurred over their career, 161/290 (55.5\%) GDPs and 47/125 (37.6\%) of specialists experienced no injuries. When the number of TNIs encountered over the past year was considered, 87.6\% GDPs reported no injuries in comparison to $76.8 \%$ specialists. Similar numbers of GDPs and specialists reported a single TNI event over their 


\begin{tabular}{|c|c|c|c|c|c|c|c|c|}
\hline \multirow[b]{3}{*}{ Number of injuries } & \multicolumn{4}{|l|}{ GDPs } & \multicolumn{4}{|l|}{ Specialists } \\
\hline & \multicolumn{2}{|c|}{ Over their career so far } & \multicolumn{2}{|c|}{ Over the past year } & \multicolumn{2}{|c|}{ Over their career so far } & \multicolumn{2}{|c|}{ Over the past year } \\
\hline & $\begin{array}{l}\text { No. of } \\
\text { clinicians }\end{array}$ & $\begin{array}{l}\text { Total no. } \\
\text { of TNls }\end{array}$ & $\begin{array}{l}\text { No. of } \\
\text { clinicians }\end{array}$ & $\begin{array}{l}\text { Total no. } \\
\text { of TNls }\end{array}$ & $\begin{array}{l}\text { No. of } \\
\text { clinicians }\end{array}$ & $\begin{array}{l}\text { Total no. } \\
\text { of TNls }\end{array}$ & $\begin{array}{l}\text { No. of } \\
\text { clinicians }\end{array}$ & $\begin{array}{l}\text { Total no. } \\
\text { of TNls }\end{array}$ \\
\hline None & 161 & - & 254 & - & 47 & - & 96 & - \\
\hline 1 & 62 & 62 & 34 & 34 & 28 & 28 & 23 & 23 \\
\hline 2 & 39 & 78 & & & 24 & 48 & 2 & 4 \\
\hline 3 & 14 & 42 & 1 & 3 & 11 & 33 & 4 & 12 \\
\hline 4 & 6 & 24 & & & 5 & 20 & & \\
\hline 5 & 5 & 25 & & & 3 & 15 & & \\
\hline$>5$ & & & & & 3 & $3 \times 6=18$ & & \\
\hline 7 & & & & & 2 & 14 & & \\
\hline 8 & 1 & 8 & & & & & & \\
\hline 10 & & & & & 1 & 10 & & \\
\hline 20 & & & & & 1 & 20 & & \\
\hline $\begin{array}{l}\text { Total number of clinicians } \\
\text { who reported TNls }\end{array}$ & 127 & & 35 & & 78 & & 29 & \\
\hline Total number & 288 & $239^{* * *}$ & 289 & 37 & 125 & 206 (minimum) & 125 & 39 \\
\hline Missing & 2 & & 1 & & & & & \\
\hline
\end{tabular}

career, at $21.4 \%$ of the GDPs compared to $22.4 \%$ of the specialists. One respondent reported an incidence of 20 TNIs within their career so far, but none in the past year. This responder was a specialist oral surgeon, who qualified in 1970. They reported that they treat about 2,000 patients a year. This may explain why they had reported a higher incidence of TNIs in comparison to others in the cohort. The LA that this responder used most often was lignocaine.

Analysis of the total number of TNIs indicated that 290 GDPs reported significantly more TNIs in their career to date than 125 specialists, at 239 compared to 206, however, the prevalence for TNIs was much higher for specialists ( $p<0.001$; Table 1) probably due to their chosen specialty involving higher risk procedures for TNIs. The total number of TNIs reported by specialists was approximate, since three specialists reported more than five injuries but did not state specific numbers.

\section{Overall prevalence of TNIs over GDP career so far}

Data obtained from 290 GDPs indicated an average of 19 years of clinical practice and a total of 239 TNIs over their career so far, which equates to almost 13 TNIs per year. This suggests $(12.58 / 290)=0.04 \mathrm{TNI}$ events per dentist per year. If every dentist has a 35-year career, it is estimated that each dentist would experience at least one TNI within their career $(35 \times 0.04=1.4)$. Extrapolating the number of TNI events per dentist per year to 22,000 active dentists within the General Dental Services (GDS), it is estimated that there are 880 TNI events/year, therefore suggesting 16.9 TNIs per week.

\section{Overall prevalence of TNIs for GDPs over the past year}

Two hundred and ninety dentists averaged 37 TNI events over the last clinical year, that is, $37 / 290=0.13$ events per dentist per year. Extrapolating this figure to the GDC-registered clinicians suggests $(0.13 \times 29,000) 3,770$ TNIs/year and 72.5 TNIs/week. If the total number of patients seen by GDPs over the year equates to approximately 54 million, this therefore equates to an incidence of approximately 14,300 procedures within the population (54,045,000 patients/3,770 TNI cases $=14,337)$.

\section{Overall prevalence of TNIs over specialist career so far}

Data obtained from 125 specialists indicated an average of 18 years of clinical practice and a minimal total of 206 TNIs over their career so far, which equates to around 11 TNIs per year. This suggests $(11.4 / 125)=0.09$ TNI events per dentist per year. If every dentist has a 35-year career, it is estimated that each dentist would experience three TNI's within their career $(35 \times 0.09=3.2)$. Extrapolating the number TNI events per dentist per year to the 800 GDC-registered specialists suggests $(0.09 \times 800) 72 \mathrm{TNIs} /$ year and 1.4 TNIs/week.

\section{Overall prevalence of TNls for specialist over the past year}

One hundred and twenty-five specialist dentists averaged 49 TNI events over the last clinical year, that is, $49 / 125=0.39$ events per dentist per year. Extrapolating this figure to the GDCregistered specialist clinicians suggests $(0.39 \times 800) 312$ TNIs/year and 6 TNIs/ week. If the total number of patients seen by specialists over the year is approximately one million, the overall incidence 
of TNI cases equates to approximately 1 in 3,300 procedures within the population $(1,026,400$ patients/312 TNI cases $=3,289)$.

The increased reporting over last year was significantly greater than reported over their career for both GDPs and specialists $(\mathrm{p}<0.001)$.

\section{Permanency of TNIs}

The majority of TNI cases encountered by GDPs were temporary $(\mathrm{n}=130 ; 68 \%)$, with $25 \%$ of cases being permanent and $7 \%$ of cases were inconclusive. The majority of TNIs reported by specialists were temporary (61\%), with 29\% being permanent. Permanency of the remaining 10\% of cases was unknown. A comparison of the permanency of the injuries reported by GDP and specialists is shown in Figure 3. Combining the TNI events reported by GDPs and specialists showed that $65.4 \%$ cases were temporary, 26.4\% were permanent and $8.2 \%$ of cases were undetermined.

Thus when comparing the rate of TNI permanency associated with low risk and high risk procedures, results indicated that more of the injuries associated with low risk procedures were temporary and more of the injuries associated with high-risk procedures were permanent ( $p<0.001$ ).

\section{Type of LA used}

When questioned about the type of LA most commonly used in their practice for inferior alveolar blocks (IANBs), lignocaine was the most commonly used LA among the GDPs and specialists (at 77\% and $91.2 \%$ respectively), followed by articaine (at 11\% and 5.6\%, respectively) (Figs 4a and b). Slightly more GDPs (9\%) than specialists (1.6\%) used mepivacaine. Other types of LA used included 3\% prilocaine, which was used by $2 \%$ of GDPs, $2 \%$ prilocaine (0.5\% of GDPs) and Citanest (0.5\% of GDPs). A small percentage of specialists $(0.8 \%)$ also used $2 \%$ prilocaine. Further analysis confirmed that there were no significant differences in the type of LA used for high-risk and low risk procedures, with lignocaine being the most commonly used LA, followed by articaine.

There were also no significant differences ( $p>0.05$ ) in the use of lignocaine between those dentists who reported multiple TNIs and those who had not encountered any within their career to date. When questioned about use of articaine as buccal

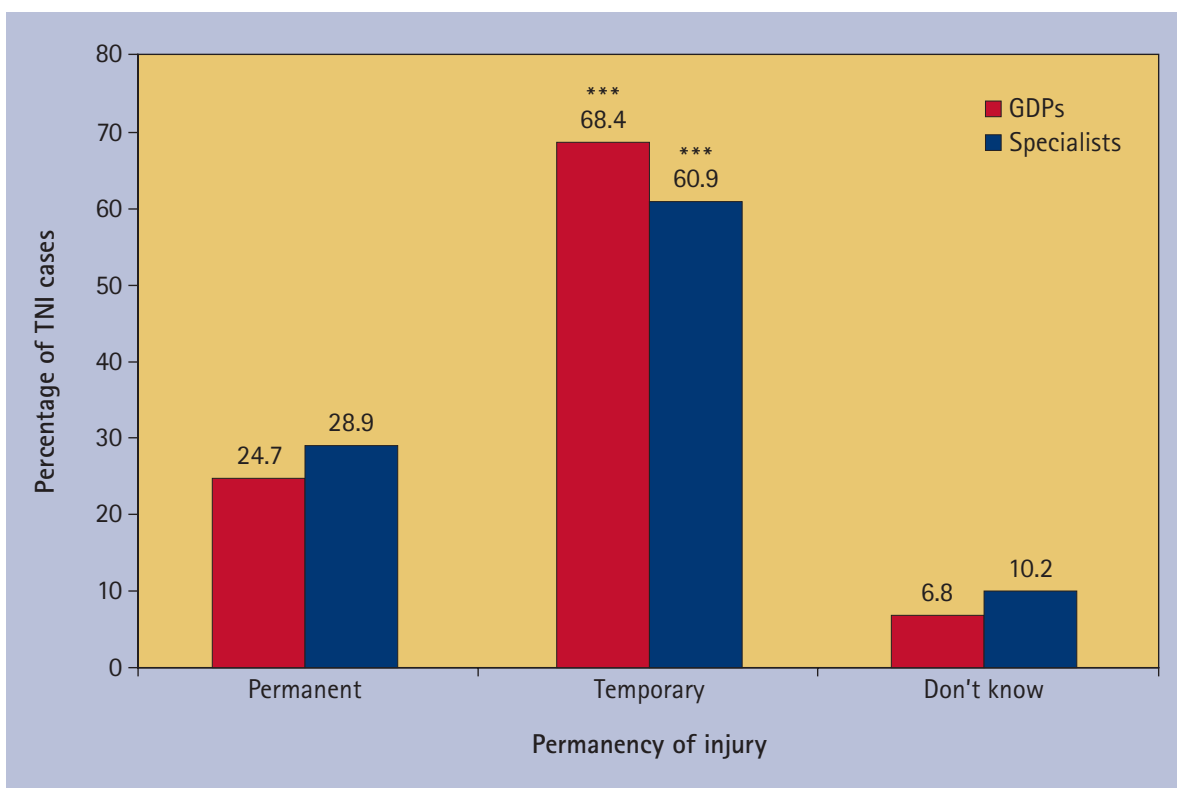

Fig. 3 A comparison of permanency of TNI's reported by GDPs and specialists, indicating that between $61 \%-68 \%$ of TNI's were temporary, which was significantly more than permanent injuries between $25 \%-29 \%\left(^{* * *}=p<0.001\right)$

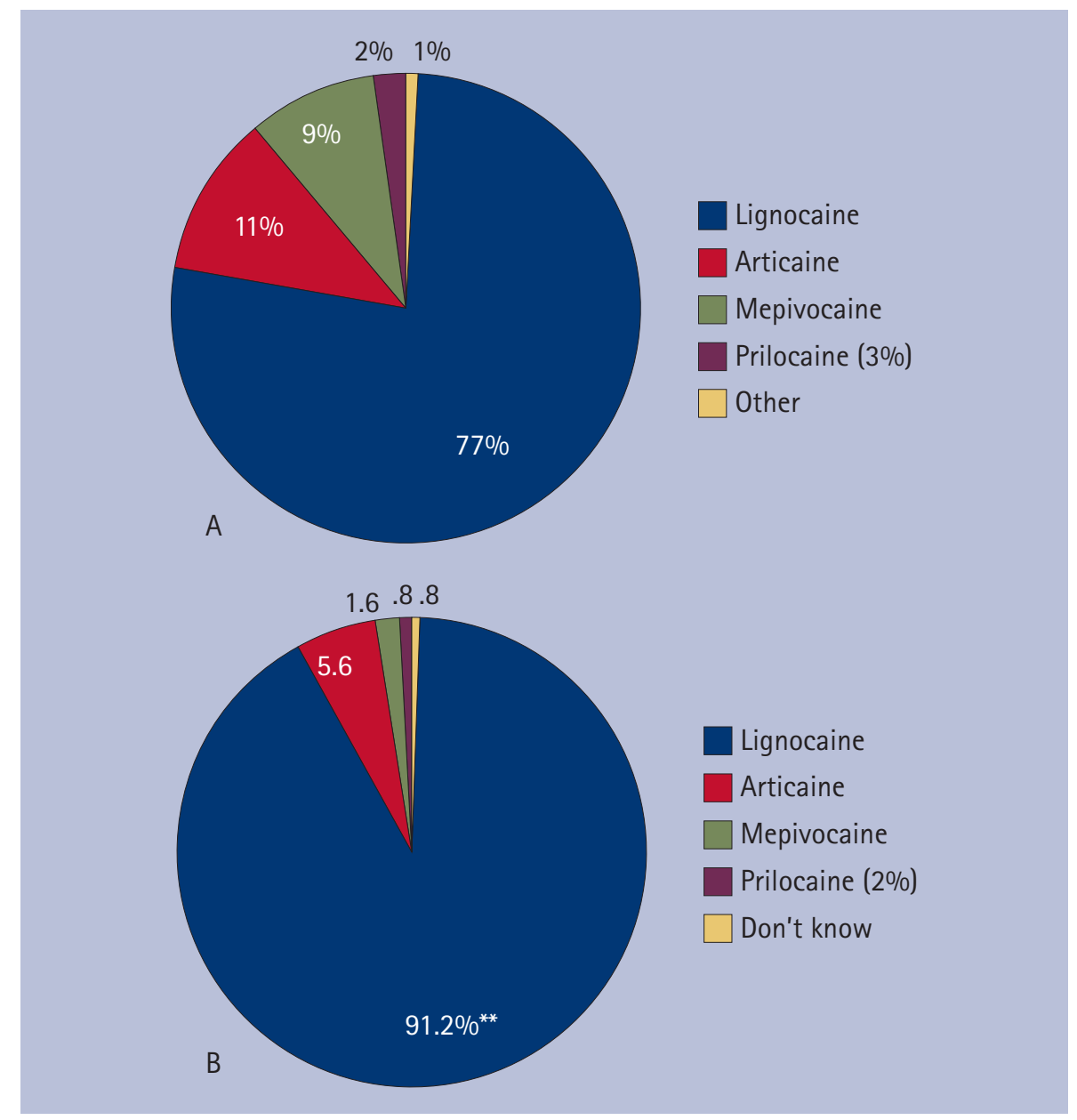

Fig. 4 Most commonly used types of LA used for ID blocks among the GDPs (a) and specialists (b): Significantly more specialists used lignocaine than the GDP's $(* *=p<0.05)$

infiltration to avoid IANBs, the responders reported that $47 \%$ of GDPs and $41.6 \%$ specialists used buccal infiltration articaine technique to avoid IANBs (8\% of GDPs did not answer this question). The other main reported indication for using articaine was repeat IANB. 


\section{Procedures associated with injuries and high-risk versus low risk procedures}

The predominant procedures associated with the TNIs among GDPs were restorative procedures when compared with third molar extractions among the OS specialists (Fig. 5). Other TNIs were associated with extraction of teeth apart from third molars, implant treatment, endodontic therapy and other procedures. In order to determine whether the injuries were due to the surgical procedure or the LA, high risk procedures were defined as third molar extractions, endodontic therapy, implant placements, apical surgery, biopsy of lip and mandibular fracture. Low risk procedures, where it was more likely that the LA was associated with the injuries, included restorative dentistry, paedodontics, periodontics and non-third molar extractions.

Almost equal percentages of TNIs reported by GDPs were associated with low $(44.8 \%)$ or high-risk procedures (50\%) over their career to date (Fig. 6a). It was not possible to know whether $12 \%$ of the cases reported by the GDPs were associated with low or high-risk procedures, as these dentists did not state this in the questionnaires. Specialists reported significantly more TNIs associated with high-risk procedures than GDPs, at $92.3 \%$ in comparison to $50 \%$ ( $p<0.01$; Fig. $6 b$ ).

\section{Post-injury care}

Post-injury care provided to the patients by both GDPs and specialists was reported most commonly to be referral to a consultant or referral to another dentist. Many practitioners reported no further care and this is likely to reflect management of patients with resolving injuries.

\section{Knowledge of where to report the injuries (Table 2)}

This question was not answered by 106 clinicians perhaps indicating a lack of awareness. Clinicians mostly reported the TNIs to medical insurance companies (23\%) and 21\% did not know where to report the injuries. Only 50\% reported the permanent injuries to various bodies including medical indemnity (over half of those reported cases), other agencies to which these cases were reported to include the medical health regulatory

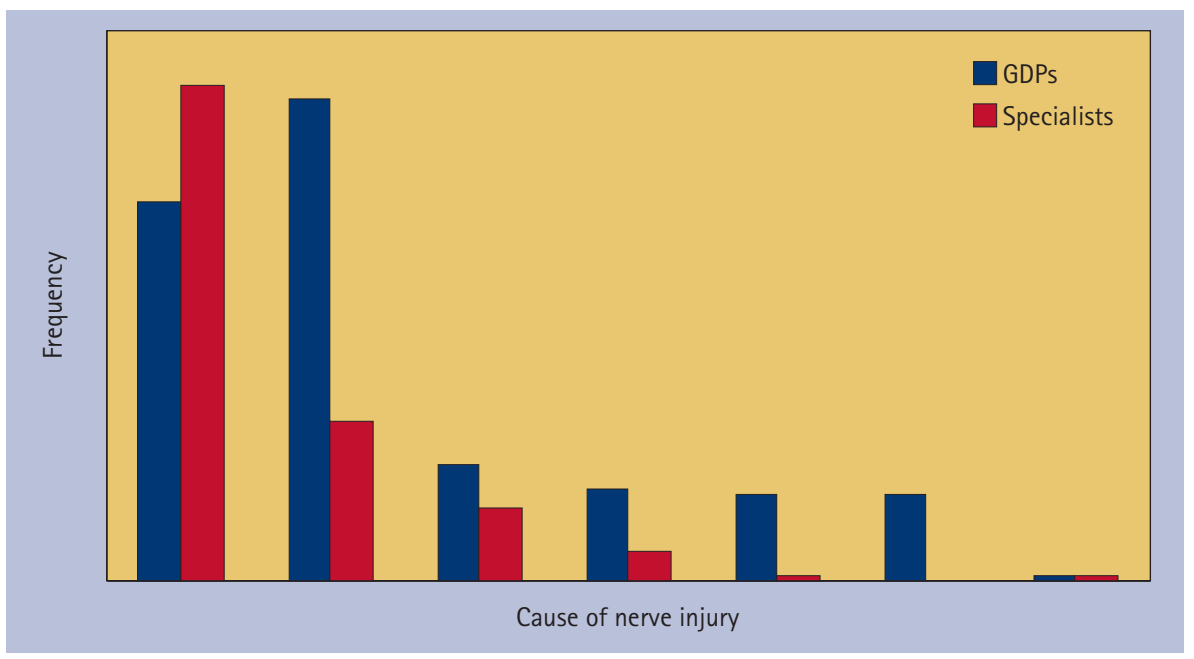

Fig. 5 Overall frequency of the reported procedures that the injuries were related to

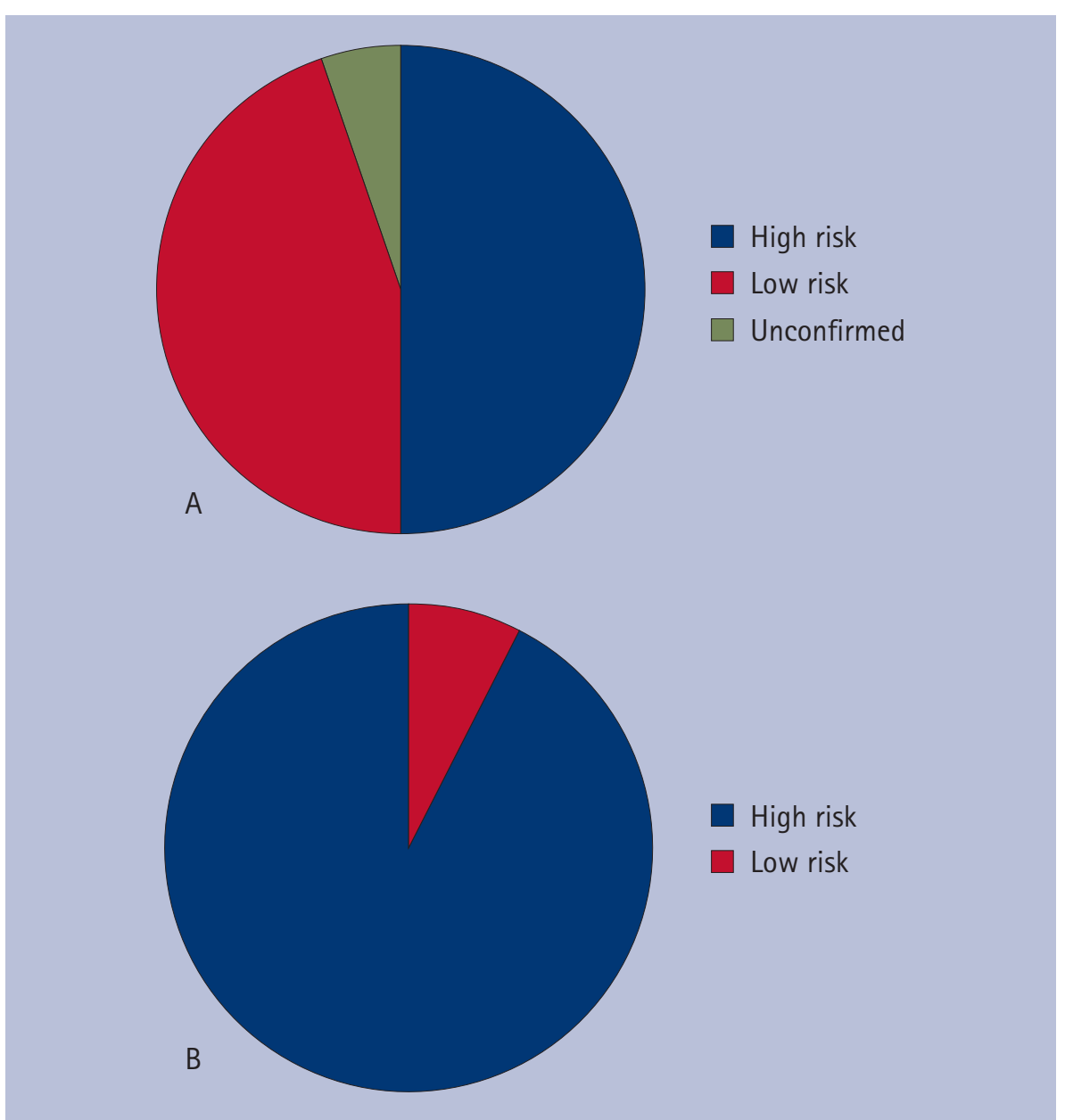

Fig. 6 Percentage of TNls associated with high risk and low risk procedures over their career, as reported by GDPs (6a; $n=290)$ and specialists (6b; $n=125)$

authority (MHRA), the local anaesthetic manufacturer and the national patient safety agency (NPSA) (Table 2). Twentyone GDPs and 12 specialists reported that they did not know where to report the injuries.

\section{DISCUSSION}

This paper provides an estimate of the volume and incidence of nerve injuries in dentistry among specialists $(\mathrm{n}=125)$ and generalists $(n=290)$. The reported activity gives a representative sample of dental procedures and activity using LA. The generalists treated more patients than the specialists even though a similar proportion of both groups were in full time practice (60\%). 


\section{Incidence of TNIs}

A majority of GDPs and minority of oral surgery specialists experienced no injuries over their careers and similar numbers of GDPs and specialists reported a single TNI event over their career (22\%), with a mean career span of 19 years for the 415 respondents.

From our data on the reported career experience of TNIs, a practising GDP would expect 0.04 TNI events per year. Assuming that every dentist has a 35-year career, it is estimated that each dentist would experience at least one TNI within their career $(35 \times 0.04=1.4)$. If we accept the current registration rate with the GDC and similar work practise, then it is estimated that there are $880 \mathrm{TNI}$ events per year, suggesting 16.9 TNIs per week occur in GDP clinics in the UK. However, based on reported experience over the last year GDPs reported a significantly higher incidence of nerve injuries with extrapolation of GDC-registered clinicians reporting 3,770 TNIs/year and 72.5 TNIs/week. This therefore equates to an incidence of approximately one TNI per 14,300 low risk procedures within the population $(54,045,000$ patients/3,770 TNI cases $=14,337)$.

The oral surgery specialists also reported a much higher incidence over the last year compared with their career experience of TNIs to date. They reported a minimal total of 206 TNIs over their 18-year career, which equates to around 11 TNIs per year or 0.09 TNI events per specialist per year. However, the specialists reported 49 TNI events over the last clinical year, extrapolating this figure to the GDC-registered specialist clinicians suggests $(0.39 \times 800) 312$ TNIs per year, 6 TNIs perweek and more than 1 TNI case per specialist per year. The overall incidence of TNI cases therefore equates to approximately 1 in 3,300 procedures within the population $(1,026,400$ patients/312 TNI cases $=3,289)$. The difference between estimates of TNI from the ten-year experience pool and last year's pool suggests that memory is a shortcoming along ${ }^{26}$ with underreporting as discussed later. Some reports may indicate that change in practice may be responsible for the recent elevated TNI rate, for example use of higher concentration LA agents. ${ }^{3}$

The prevalence for TNIs was much higher for specialists compared with generalists

\begin{tabular}{|c|c|c|}
\hline & Frequency & Percentage \\
\hline Not answered & 106 & 52.2 \\
\hline Medical insurance & 47 & 23.2 \\
\hline Does not know & 21 & 10.3 \\
\hline MHRA & 5 & 2.5 \\
\hline MHRA and medical insurance & 4 & 2.0 \\
\hline NPSA & 4 & 2.0 \\
\hline MHRA, LA manufacturer and medical insurance & 3 & 1.5 \\
\hline NPSA and medical insurance & 3 & 1.5 \\
\hline LA manufacturer and medical insurance & 3 & 1.5 \\
\hline Other & 3 & 1.5 \\
\hline LA manufacturer & 2 & 1.0 \\
\hline NPSA and other & 1 & 0.5 \\
\hline ROIA & 1 & 0.5 \\
\hline
\end{tabular}

( $p<0.001$; Table 1) probably due to their chosen specialty involving higher risk procedures for TNIs.

Based on these results of this study we estimate that the incidence of LA-related nerve injuries is significantly higher than previously reported. ${ }^{3,4,7}$ For example, it is estimated that 1 in 3,300 TNIs will occur for high risk procedures such as third molar surgery. This ratio is much less at 1 in 14,300 for low risk procedures, such as restorative dentistry.

In dentistry we are not obliged to warn the patient of these injuries as we are taught at dental school that the incidence is less than 1:500,000, thus extremely rare. In an opinion article from Hillerup S et al. ${ }^{5}$ it has been extrapolated that the incidence is likely to be higher than this: for example, an average NHS dentist working for 25-30 years probably administers at least 100,000-150,000 inferior dental blocks (IANBs). Interestingly a recent national UK audit by the Royal College of Anaesthetists ${ }^{27}$ reports the estimated nerve injury resulting from neuroaxial blocks (epidurals, spinals and combined epidural with spinals) resulted in sensory or motor nerve injury in 1 in 24,000-54,000 patients (and paraplegia or death in 1 in 50,000-140,000 patients). UK anaesthetists are obligated to routinely warn their patients regarding potential permanent nerve injury when using these techniques with a significantly lower incidence of iatrogenic nerve injury risk compared with dentists.

\section{Permanency of TNIs}

The majority of TNI cases reported by GDPs and specialists were temporary (68\% or $61 \%$ respectively), with $25-29 \%$ being reported as permanent. This seems a high rate of permanency compared with previous reports. ${ }^{24}$ One may question as to whether this relates to the TNIs in this study being related to low risk procedures and thus most likely LA-related. Evidence suggests that lingual nerve injuries in relation to lingual access third molar surgery are predominantly temporary, ${ }^{28,29}$ however, little is known of the prognosis of inferior alveolar nerve injuries in relation to various dental interventions.

These data suggest that Specialists are more likely to encounter permanent TNIs than GDPs again probably based upon the high risk nature of their specialist procedures as more of the injuries initiated by high-risk procedures were permanent (p <0.01) (Fig. 6).

\section{Type of LA used}

The most common LA agent used for inferior alveolar blocks (IANBs) was lignocaine, interestingly more so by specialists (91\%) than GDPs (77\%) (Fig. 4). Articaine was the second most popular choice of LA more commonly used by GDPs compared with specialists, $11 \%$ and $5.6 \%$ respectively. GDPs were more likely to use alternative LA agents in 25\% of cases including mepivacaine, prilocaine and Citanest. Further analysis confirmed 
that there were no significant differences in the type of LA used for high risk and low risk procedures, with lignocaine being the most commonly used LA, followed by articaine.

Interestingly there was no significant difference in the use of lignocaine between those dentists who reported multiple TNIs and those who had not encountered any within their career to date ( $p>0.05$ ). When questioned about use of articaine as buccal infiltration to avoid IANBs the responders reported that $47 \%$ of GDPs and $41.6 \%$ specialists used this technique to avoid IANBs (8\% of GDPs did not answer this question).

Of interest was that many practitioners are already reporting using articaine infiltrations instead of IANBs to provide local anaesthesia (45.6\%) even though there is limited evidence base for this practice. ${ }^{30}$ Articaine is reported to be efficient in pain relief during and post apicectomy procedures using conventional IAN block injections. ${ }^{31}$ Buccal infiltration techniques as an alternative to IANBs have been suggested for implant surgery ${ }^{32}$ and it is becoming routine practice for orthodontic extraction of premolars and restorative treatment of premolars and molars in adults. ${ }^{33}$ Interestingly more recently articaine infiltrations are demonstrating similar efficacy to lignocaine IANBs for mandibular dentistry therefore removing the necessity of an IANB completely. ${ }^{34}$ It has become routine practice for paedodontic extraction of premolars using articaine infiltrations and many practitioners are routinely undertaking restorative treatment of premolars and molars in adults using LA infiltrations rather than IANBs. It is likely that this new practice could significantly reduce the incidence of dental LA TNIs.

Persistent sensory neuropathy or nerve pain associated with articaine IANBs for routine dentistry has been reported. ${ }^{3,4,20,35-41}$ However, a double crossover study assessed the comparative efficiency of articaine versus lignocaine reported no significant difference in onset of action and pain experience after buccal and palatal infiltrative injections using 4\% articaine $1: 100,000$ adrenaline or $2 \%$ lignocaine $1: 100,000$ adrenaline. ${ }^{39}$ The product information sheets on the Septodont website (www.septodont.co.uk/Articaineuk/prescribing/spc1hundred.html) state that resolution usually takes place within two weeks, which does not fit with the findings of this study.

\section{Procedures associated with the injuries and high risk versus low risk procedures}

The predominant procedures associated with initiation of the TNIs among the GDPs were restorative procedures, compared to third molar extractions among the OS specialists (Fig. 5). Other TNIs were associated with extraction of teeth apart from third molars, implant treatment, endodontic therapy and other procedures. In order to determine whether the injuries were due to the surgical procedure or the LA, high risk procedures were defined as third molar extractions, endodontic therapy, implant placements, apical surgery, biopsy of lip and mandibular fracture. The low risk procedures included restorative dentistry, paedodontics, periodontics and non-third molar extractions; these were deemed that it was more likely that the LA was associated with the nerve injuries. This strategy is an oversimplified separation of aetiology of nerve injury based upon technique as LA may have been associated with the nerve injuries in some of the high risk procedures. However, as a result of this division of techniques into high and low risk, the incidence of LA-related nerve injuries will be underestimated rather than overestimated.

Almost equal percentages of TNIs reported by GDPs were associated with low (44.8\%) or high-risk procedures (50\%) over their career to date (Fig. 6a). Whereas specialists reported significantly more TNIs associated with high-risk procedures than GDPs, at $92.3 \%$ in comparison to $50 \%$ $\mathrm{p}<0.01$; Fig. $6 \mathrm{~b})$. This most likely reflects the high risk nature of procedures undertaken by specialists, being more complex with inherent higher risk for nerve injury.

\section{Prevention}

For specialists, the most common procedure associated with the nerve injury was third molar extraction and for GDPs it was restorative (or LA related), as indicated in Figure 5. LA nerve injuries can be minimised based upon previously identified risk factors, ${ }^{23}$ which include high concentration LA IANBs, ${ }^{3,4,7,23,35}$ multiple IANBs and pain on injection. ${ }^{6,23}$ The buccal surgical approach significantly reduces lingual nerve injuries in relation to third molar extraction nerve injuries..$^{42}$ Inferior alveolar nerve injuries can be avoided by using preoperative assessment of high risk third molars using Cone Beam CT where required and the coronectomy procedure when indicated. ${ }^{43}$

\section{Reporting}

Clinicians in this study only reported their known nerve injury cases in 50\% of cases and then mostly to their indemnity insurance companies. Unfortunately this practice does not provide any national data on incidence risk factors of these nerve injuries thus minimising the potential to inform practice and thus prevent these injuries. It is recommended that clinicians should document unusual patient reactions occurring during the injection of LA blocks (such as sharp pain or an electrical shocklike sensation) and at least on those occasions check on their patient recovery from LA post-surgically, improving patient care.

There appears to be some considerable confusion as to where to refer these patients with nerve injuries. Dentists most commonly referred patients with persistent LA nerve injuries to a consultant, or another dentist which may, or may not, be appropriate. If the clinician was better informed local management and referral to appropriate websites for patient and clinician advice would be optimal. ${ }^{22}$

Clear guidelines now exist from the Care Quality Commission, whereby if a treatment related nerve injury persists longer than 28 days post-surgery the dentist or registered practice manager are obligated to report the complication to the CQC. ${ }^{44}$ The patient is also able to self-report their nerve injury. The LA manufacturers should also have an easier and more transparent process to report these complications and provide warnings in the drug literature of the possibility of permanent nerve injury.

\section{Management}

Recommendations suggest that if a peripheral sensory nerve injury is suspected the nerve should be immediately explored and repaired, however, this is not applicable to LA nerve injuries. Management of TNIs related to dentistry is complex. The 'sit and wait' policy can be applied to lingual nerve injuries related to third molar surgery, LA and complex surgery 
(cancer, orthognathic, fractures). But all other injuries including inferior alveolar nerve injury related to implants, endodontics and third molar surgery require more urgent addressing, at least within 30 hours. Surgery is only indicated in relatively few patients and neuropathic pain, often experienced in relation to theses TNIs, does not respond to surgical intervention. If the patient develops chronic pain then chronic pain medication and possible psychological therapies may be indicated. ${ }^{22,23}$ Management in the acute phase lacks evidence base and early prescription of high dose steroids and or NSAIDs may facilitate a reduction in neural inflammation.

The clinician must not overlook the distress and complications related to TNIs when they occur, no matter how they are caused. The patient deserves care, consideration and recognition of their injury with appropriate advice. Better information about the potential incidence of these injuries is required and an improved understanding of how to prevent LA nerve injuries is imperative as they cannot be 'cured'.

\section{ISSUES WITH STUDY DESIGN}

In order to assess the incidence of dental procedural-related trigeminal nerve injury we had several possible options. One of these included obtaining the GDC list of GDPs to mailshot them. Another included assessing the total number of UK referrals of patients to Sheffield and London nerve injury referral services. Another alternative was to assess hospital episode statistics (HES) data for trigeminal nerve repair, or to examine existing sources of data, such as the King's College patient database, MHRA Yellow Card database, Insurance companies' databases, dentists' existing records, or other (unknown) dentist surveys.

Whatever method or sourced sample we elected to choose would include an element of bias; for example more willingness to take part in a survey or compliance with reporting mechanisms. Even a wholesale questionnaire to all dentists and/or patients is unlikely to produce much more than a $50 \%$ return rate. The GDC listings are not openly available for accessing dentists and the addresses are often incorrect due to younger dentists using their parents' addresses for example. The error may be systematic, for example dentists with a high rate of injuries may obviously tend not to reply or reply honestly and most normal dentists can be expected to 'forget' undesired outcomes, either intentionally or due to the passage of time. We accepted that whatever method we adopted there would be attendant significant errors. It may be the error rate due to these factors is the dominant effect over sampling error even with a small sample. This suggests that a focus on a small representative sample very carefully controlled might be just as effective as a large scale survey with little control.

Thus with the method we used, that is, the anonymised questionnaire presented to dentists attending CPD days on oral surgery developments throughout the UK and specialists attending the British Association of Oral Surgeons conference, we accepted inherent bias. The sample was biased towards oral surgery specialists, towards dentists actively registering and attending CPD meetings and those willing to provide anonymised information at the meeting.

Sampling and bias issues arising with our chosen method were three-fold. The sample of dentists was self-selecting from conference attendees. It is possible such a grouping would have a lower incidence of LA-related TNIs because conference attendees are likely to be more concerned about training and skills. Those with high incidence of nerve injury rates might, for that reason, elect not to take part. Alternatively they could have been more interested in the survey because they have experienced nerve injury.

Those completing the questionnaire might under-report because they genuinely forgot incidents, especially for multiple occurrences. However, on the other hand they may intentionally forget due to guilty feelings or liability concerns, again especially for multiple incidents. All reports depend on patient interaction. Some patients, in particular those less demanding or those more stoical, may not report incidents, thus the rates above are a likely understatement of the true rates of LA related TNI.

\section{DESIGN ISSUES WITH THE OUESTIONNAIRE}

We identified some weaknesses in the questionnaire design, which included the lack of space for dentists to report more than five events. This means that of the 315 events reported, 32 could not be reported as permanent or temporary, resulting in a deficiency in detailed reporting of the permanent injuries.

\section{ISSUES ARISING REQUIRING FURTHER INVESTIGATION}

Thirty percent of clinicians had not experienced LA-related TNI across an average career experience of over 18 years and more recently qualified dentists reported less injuries compared with experienced clinicians, which was probably related to patient numbers. The dentists who experienced exceptionally high rates of nerve injury (8-20 cases) had been qualified for 20-43 years. However, some less experienced clinicians reported a high incidence of TNIs. This raises the question of whether these clinicians require additional scrutiny and/or training.

Most of these TNIs occurred during restorative treatment and is an unforeseen and unpleasant complication for patients. Due to the incidence of nerve injuries in relation to dental anaesthesia, warning of patients is not considered necessary and in the UK these iatrogenic injuries are not considered to be negligent. However, anaesthetists are obliged to warn their patients of a 1 in 200,000 risk of sensory or motor nerve injuries in relation to spinal block injections.

Many clinicians would argue that any additional warnings of risks would contribute to further patient anxiety which is not ideal; however, better information about the possibility and consequences of these injuries may ensure better practice and improved patient ability to cope with these injuries.

Lastly, since LA-related TNI appears to be the most common adverse effect from LA, and the rate appears to be significantly higher than previously reported, would it be of public benefit for pharmaceutical companies to provide preventative advice for dentists in the packaging? This is definitely a question worth further debate and investigation.

\section{CONCLUSION}

Based on the data presented, it appears that trigeminal nerve injuries in relation to dental procedures, particularly LA, are 
significantly more frequently encountered by UK dentists than previously thought. Approximately a third of these nerve injuries are reported to be permanent; again a higher figure than previously published. Most of the injuries in this survey were related to restorative practice using lignocaine IANBs. A significant number of clinicians are already minimising the risk of IANB related nerve injury by using higher concentration LA infiltrations for routine dentistry.

As a result of our study on TNI related to dentistry as described in this paper, we recommend that practitioners adopt preventive strategies to avoid dentistry-related TNIs. For example with regard to LA-related TNIs dentists should consider the use of high concentration buccal infiltrations to replace mandibular block anaesthesia. Most practitioners surveyed in this study are using 2\% lignocaine for IANBs and we support this practice. Patients having local anaesthesia should each be advised to report back to the surgery if any numbness/tingling/pain persists for more than 48 hours. If implemented, this procedure opens the way for early identification of nerve injuries and reassurance of the patients involved. This will also enable the clinician to accurately monitor and report appropriate injuries to the $\mathrm{CQC}$, allowing an accurate assessment of the prevalence of these LA-related nerve injuries and thus inform better practice to prevent these injuries. Further information can be found at http://trigeminalnerve.org.uk/.

We would like to thank Mike Dalgleish for his generosity in sponsoring the project through the 'Trigeminal Foundation'.

1. Renton T, Yılmaz Z. Profiling of patients presenting with posttraumatic neuropathy of the trigeminal nerve. J Orofacial Pain 2011; 25: 333-344.

2. Pogrel M A, Thamby S. Permanent nerve involvement resulting from inferior alveolar nerve blocks. J Am Dent Assoc 2000; 131: 901-907.

3. Haas D A, Lennon D. A 21 year restrospective study of reports of paraesthesia following local anaesthetic administration. J Can Dent Assoc 1995; 61: 319-330.

4. Hillerup S, Jensen R. Nerve injury caused by mandibular block analgesia. Int J Oral Maxillofac Surg
2006; 35: 437-443.

5. Hillerup S, Jensen R H, Ersbø\|l B K. Trigeminal NI associated with injection of local anesthetics: needle lesion or neurotoxicity? J Am Dent Assoc 2011; 142: 531-539.

6. Robinson P P. Characteristics of patients referred to a UK trigeminal nerve injury service. J Oral Surg 2011; 4: 8-14.

7. Hillerup $S$. latrogenic injury to oral branches of the trigeminal nerve: records of 449 cases. Clin Oral Investig 2007; 11: 133-142.

8. Pogrel M A, Schmidt B L, Sambajon V, Jordan R C. Lingual nerve damage due to inferior alveolar nerve blocks: possible explanation. J Am Dent Assoc 2003; 134: 195-199.

9. Harn S D, Durham T M. Incidence of lingual nerve trauma and post injection complications in conventional mandibular block anesthesia. J Am Dent Assoc 1990; 121: 519-523.

10. Perez-Castro R, Patel S, Garavito-Aguilar Z V et al. Cytotoxicity of local anesthetics in human neurona cells. Anesth Analg 2009; 108: 997-1007.

11. Loescher A R, Robinson P P. The effect of surgical medicaments on peripheral nerve function. Br J Oral Maxillofac Surg 1998; 36: 327-332.

12. Pogrel M A. Summary of: Trigeminal nerve injuries in relation to the local anaesthesia in mandibular injections. Br Dent J. 2010; 209: 452-453.

13. Riberio P D Jr, Sanches M G, Okamoto T. Comparative analysis of tissue reactions to anaesthetic solutions using histological analysis in subcutaneous tissues of rats Anaesth Prog 2003; 50: 169-180

14. Oertel R, Rahn R, Kirch W. Clinical pharmacokinetics of Articaine. Clin Pharmacokinet 1997; 33: 417-423.

15. Brandt R G, Anderson P F, McDonald N J, Sohn W, Peters M C. The pulpal anesthetic efficacy of Articaine versus Lignocaine in dentistry: a metaanalysis. J Am Dent Assoc 2011; 142: 493-504.

16. Simon M A, Vree T B, Gielen M J, Booij M H Comparison of the effects and disposition kinetics of Articaine and Lignocaine in 20 patients undergoing intravenous regional anaesthesia during day case surgery. Pharm World Sci 1998; 20: 88-94.

17. Mikesell P, Nusstein J, Reader A, Beck M, Weaver J. A comparison of Articaine and Lignocaine for inferior alveolar nerve blocks. J Endod 2005. 31: 265-270.

18. Huang Y D, Xia H, Li X D, Yang X Z, Pei Z O, Xia X A comparison of the clinical anesthetic efficacy of Articaine infiltration and Lignocaine blocking for microport extraction of impacted mandibular molar. Hua Xi Kou Qiang Yi Xue Za Zhi 2011; 29: 268-271.

19. Cornelius $C P$, Roser $M$, Wiethölter $H$, Wolburg $H$. Nerve injection injuries due to local anaesthetics. Experimental work. J Cranio Max Fac Surg 2000; 28(Suppl 3): 134-135.

20. Van Eeden S P, Patel M F. Prolonged paraesthesia following inferior alveolar nerve block using articaine. Br J Oral Maxillofac Surg 2002; 40: 519-520.

21. Hillerup S, Bakke M, Larsen J O, Thomsen C E, Gerds T A. Concentration-dependent neurotoxicity of Articaine: an electrophysiological and stereological study of the rat sciatic nerve. Anesth Analg 2011 112: 1330-1338.

22. Renton T, Yilmaz Z. Managing iatrogenic trigeminal nerve injury: a case series and review of the literature. Int J Oral Maxillofac Surg 2012; 41: 629-637.

23. Renton T, Adey-Viscuso D, Meechan J G, Yilmaz Z. Trigeminal nerve injuries in relation to the local anaesthesia in mandibular injections. Br Dent J
2010; 209: E15.

24. Smith $M H_{\text {, Lung } K}$ E. Nerve injuries after dental injection: a review of the literature. J Can Dent Assoc 2006; 72: 559-564.

25. Health and Social Care Information Centre, Prescrbing and Primary Care. NHS dental statistics for England: 2011/12. London: Health and Social Care Information Centre, 2012

26. Hazell L, Shakir S A. Under-reporting of adverse drug reactions: a systematic review. Drug Saf 2006 29: 385-396.

27. NAP 3: report and findings of the 3rd National Audit Project of the Royal College of Anaesthetists. Ed Dr Tim Cook NAP3 Lead. Royal College of Anaesthetists, 2009. Online report available at http://www.rcoa.ac.uk/nap3 (accessed May 2013).

28. Mason D A. Lingual nerve damage following lower third molar surgery. Int J Oral Maxillofac Surg 1988; 17: 290-294.

29. Blackburn C W, Bramley P A. Lingual nerve damage associated with the removal of third molars. $\mathrm{Br}$ Dent J 1989; 167: 103-107.

30. Malamed S F, Gagnon S, Leblanc D. Articaine hydrochloride: a study of the safety of a new amide local anesthetic. J Am Dent Assoc 2006; 132: 177-185.

31. Meechan J G, Blair G S. The effect of two different local anaesthetic solutions on pain experience following apicectomy. Br Dent J 1993; 175: 410-413.

32. Heller A A, Shankland WE 2nd. Alternative to the inferior alveolar nerve block anesthesia when placing mandibular dental implants posterior to the mental foramen. J Oral Implantol 2001: 27: 127-133.

33. Corbett I P, Kanaa M D, Whitworth J M, Meechan $J$ G. Articaine infiltration for anesthesia of mandibular first molars. J Endod 2008; 34: 514-518.

34. Kanaa M D, Whitworth J M, Corbett I P, Meechan $J$ G. Articaine buccal infiltration enhances the effectiveness of Lignocaine inferior alveolar nerve block. Int Endod J 2009; 42: 238-246.

35. Haas D A. Articaine and paresthesia: epidemiological studies. J Am Coll Dent 2006; 73: 5-10.

36. Krafft T C, Hickel R. Clinical investigation into the incidence of direct damage to the lingual nerve caused by local anaesthesia. J Craniomaxillofac Surg 1994; 22: 294-296.

37. Wyman R J. Nerve injury following a mandibular block: a case report. Dent Today 2010; 29: 14.

38. Stacy G C, Hajjar G. Barbed needle and inexplicable paresthesias and trismus after dental regional anaesthesia. Oral Surg Oral Med Oral Pathol 1994; 77: 585-588.

39. Oliveira P C, Volpato M C, Ramacciato J C, Ranali $J$. Articaine and lignocaine efficiency in infiltration anaesthesia: a pilot study. Br Dent J 2004; 197: 45-46.

40. Kirihara $Y$, Saito $Y$, Sakura S, Hashimoto $K$ Kishimoto T, Yasui Y. Comparative neurotoxicity of intrathecal and epidural Lignocaine in rats. Anesthesiology 2003; 99: 961-968.

41. Crean S J, Powis A. Neurological complications of local anaesthetics in dentistry. Dent Update 1999 26: 344-349

42. Robinson P P, Loescher A R, Smith K G. The effect of surgical technique on lingual nerve damage during lower 3rd molar removal by dental students. Eur J Dent Educ 1999; 3: 52-55.

43. Renton T. Prevention of iatrogenic inferior alveolar nerve injuries in relation to dental procedures. Dent Update 2010; 37: 350-363.

44. CQC statutory notifications Statutory notificationsCare Quality Commission http://www.cqc.org.uk/ organisations-we-regulate/registered-services/ notifications. 